\title{
Significance of adenosquamous proliferation in breast lesions
}

\author{
Mark James Wilsher
}

\section{Correspondence to}

Dr Mark James Wilsher,

Histopathology, University Hospital Lewisham, London SE136LH, UK; markwilsher@ gmail.com

Received 8 September 2020 Revised 27 November 2020 Accepted 7 December 2020 Published Online First 30 December 2020

Check for updates

(c) Author(s) (or their employer(s)) 2021. No commercial re-use. See rights and permissions. Published by BMJ.

To cite: Wilsher MJ.

J Clin Pathol

2021:74:559-567.

\begin{abstract}
Adenosquamous proliferation (ASP), characterised by ductal structures with a dual glandular and squamous phenotype within desmoplastic stroma, is essentially a hallmark of various sclerosing lesions of the breast (SL) and breast lesions with sclerosis (BLWS), not including sclerosing adenosis. In radial scar/complex sclerosing lesion (RS/CSL), clonality has been previously demonstrated in microdissected ASP. SL/BLWS encompass a diverse range of pathological entities that historically have an equally diverse list of names, often for histologically alike or identical lesions at different anatomical locations. In common they are comprised of one or more components of fibrocystic or proliferative breast disease and papillomata, which become distorted and even obliterated by a sclerosing process that appears to be associated with and/or secondary to ASP, which in an individual lesion may be inconspicuous at the time of biopsy. The histological overlap of various SL/ BLWS with RS/CSL, in which a nidus containing ASP is pathognomonic of early lesions, also supports a common element of ASP across various SL/BLWS. SL/BLWS show an interesting association with low-grade metaplastic carcinoma, particularly low-grade adenosquamous carcinoma (LGASC) with which, they appear to form a histological and possible biological spectrum because ASP and LGASC share similar histological and immunophenotypical characteristics. The presentation of ASP in various SL/BLWS will be discussed.
\end{abstract}

\section{INTRODUCTION}

Adenosquamous proliferation (ASP) of the breast ${ }^{1}$ is a lesion characterised by compact ducts with both glandular and squamous features within a variable spindle cell stroma. They are most commonly encountered in the context of various sclerosing breast lesions (SL), or in subsets of benign entities that may show sclerosis (breast lesions with sclerosis (BLWS)), for example, intraduct papilloma (IP) and nipple adenoma (NA). Historically, various nomenclatures have been used for histologically alike, to essentially identical SL/BLWS at different anatomical locations. They all similarly occur at a broad age range. Histologically, they share in common variable degrees of proliferative, fibrocystic and papillomatous change and a variable content of ASP. SL/BLWS often show temporal variability, for example, in radial scar/complex sclerosing lesion (RS/CSL), an early and late phase is typical. They may mimic carcinoma-clinically, radiologically and histologically and indeed also show an association with epithelial atypia, in-situ and invasive mammary carcinoma. SL/BLWS show an interesting association with low-grade metaplastic carcinoma, particularly low-grade adenosquamous carcinoma (LGASC) with which, they seem to form a histological and possible biological spectrum. ${ }^{1}$ The distinction of a SL/BLWS, for example, RS/CSL with prominent ASP and LGASC can be challenging. ${ }^{12}$

$\mathrm{RS} / \mathrm{CSL}$ is probably the most common SL encountered by pathologists and one which historically has the most diverse list of names. ${ }^{3-17}$ Micropapillomata are not infrequently seen in RS/CSL, but when sclerosing changes occur in predominant papillomata they are termed 'sclerosing papilloma (SP)', which is related to the lesion termed 'ductal adenoma (DA)' introduced by Azzopardi and Salm. ${ }^{18}$ Infiltrating epitheliosis (IE), currently not a WHO recognised entity, is considered to lie within the spectrum of RS/ CSL. It shows a predominance of florid usual ductal hyperplasia (UDH) and/or a UDH-like process. The 'pattern' may form a histological continuum with the newly WHO recognised entity, tall cell carcinoma with reversed polarity (TCCRP). ${ }^{19-21} \mathrm{NA}$ (florid papillomatosis (FP) of the nipple) ${ }^{22}$-the sclerosing papillomatous variant, and the deeper variant-subareolar sclerosing duct hyperplasia $(\mathrm{SSDH}),{ }^{23}$ may show histological features overlapping with RS/CSL, SP and DA. Syringomatous tumour (ST) resembles an LGASC confined to the nipple, the latter typically presenting within deeper and peripheral breast tissue, but of which, has also been diagnosed in a pure nipple location ${ }^{24}$ and the two indeed may represent the same lesion. ${ }^{25}$ They have a dual squamous and glandular phenotype and closely resemble the ASP seen in the context of SL/ BLWS, but the latter is the predominant histological feature.

The aetiology of RL/CSL (and related SL) has been debated over time, but most recently it is considered that they may represent non-obligate clonal, neoplastic precursor lesions. ${ }^{26-28}$

The nature of ASP and its appearance in various SL/BLWS will be discussed.

\section{ADENOSQUAMOUS PROLIFERATION}

ASP is an epithelial proliferation with a dual glandular and squamous phenotype, typically within a variable spindle cell (desmoplastic) stroma forming a lamellar cuff, and often with a syringoid appearance. ASP often appears to stream-out from or peel-off pre-existing ducts. It is not recognised as a specific entity by the WHO, yet it is essentially a hallmark of SL/BLWS, but may rarely be seen in other settings such as fibroepithelial lesions. The appearance is essentially histologically and immunophenotypically the same as the neoplastic 
proliferation characteristic of LGASC. ${ }^{1}$ Indeed, using next generation sequencing (NGS) of microdissected ASP from RS/CSL, it has been shown to be a clonal proliferation in that context at least. ${ }^{27}$ This may help explain the association of various SL/ BLWS with LGASC and also the challenges in distinguishing such lesions with prominent ASP from an LGASC. ${ }^{127}$ LGASC is commonly reported in association with or emanating from benign proliferative and SL such as RL/CSL (including IE), IP, adenomyoepithelioma and NA. ${ }^{24} 2629-33$

In common with LGASC (and ST), ASP is triple negative for oestrogen and progesterone receptors and HER2, and displays coexpression of high-molecular weight cytokeratins such as cytokeratin 5/6 and p63. It lacks morphologically and phenotypically normal myoepithelial cells, showing their inconsistent presence, and is typically surrounded by reactive myofibroblasts, which express myoid immunomarkers but do not coexpress p63 as would myoepithelial cells. Therefore, immunohistochemistry cannot be used to distinguish a SL/BLWS rich in ASP and LGASC. Their distinction is typically based on the extent of ASP and degree of infiltration into surrounding breast tissue. ASP at least in the context of an RS/CSL appears to regress and disappear over time ('burns out'), being characteristic of early RS/ CSL and diminished to absent in late RS/CSL where the nidus is replaced by fibroelastosis. ${ }^{1}$ Suster $e t a l^{34}$ described four cases of 'syringomatous squamous tumours of the breast' ranging from $0.5 \mathrm{~cm}$ to $1.5 \mathrm{~cm}$, which resembled LGASC, that they theorised may represent a preneoplastic or low-grade neoplastic process and a possible precursor stage of LGASC.

\section{RS/CSL INCLUDING SO-CALLED IE}

RS is perhaps the most recognisable term for this relatively frequently encountered SL. RS is a translation of 'strahlige narben' in German used by Hamperl ${ }^{3}$ in 1975 due to its stellate architecture. The term 'proliferation centre' (of Aschoff) is attributed to Ludwig Aschoff. ' However, 'proliferative growth centres' were described by Schaper and Cohen in a 1905 publication as a general embryological and pathological concept not specific to breast ${ }^{8}$ and Aschoff's textbook ${ }^{9}$ later referenced that publication. Numerous epithets exist (figure 1). ${ }^{3-17}$

RS have a characteristic floral architecture, with ducts radiating from a central nidus out to a peripheral corona with a rosette-type appearance, whereas CSL may be more disorganised and sometimes nodular in appearance, and can be formed of merging adjacent smaller lesions. ${ }^{1635}$ The nidus (centre) contains ASP at its inception, with fibroelastosis inversely proportional to the amount of ASP which reduce/involute over time. ${ }^{1}$ It typically arises within an area of hyperplasia, fibrocystic change \pm micropapillomata, which forms its corona. CSL shows a greater disturbance of architecture and may include papilloma formation, apocrine metaplasia and sclerosing adenosis. ${ }^{1635}$ The ASP appears to essentially distort the latter and to consequently induce the stellate/distorted architecture. ${ }^{127}$ The overall incidence is $4.7 \%-$ $8.2 \% .^{26}$ The incidence on needle core biopsy (NCB) is $1 \%-2 \%{ }^{36}$

A zone of stromal and periductal elastosis is seen as pathognomonic of an RS/CSL and is indeed a key component of the nidus in late lesions. However, this is a secondary, degenerative feature after an initial proliferative phase, along with fibrous sclerosis. Elastosis is not unique to an RS/CSL and its presence may lead to over diagnosis of the latter. Elastosis is seen in various benign breast diseases (eg, duct ectasia and fibrocystic disease) and is common in carcinoma. ${ }^{12} 37$ Parfrey and Doyle ${ }^{38}$ found that in fibrocystic disease, stromal and periductal elastosis were related to the degree of epitheliosis (UDH).

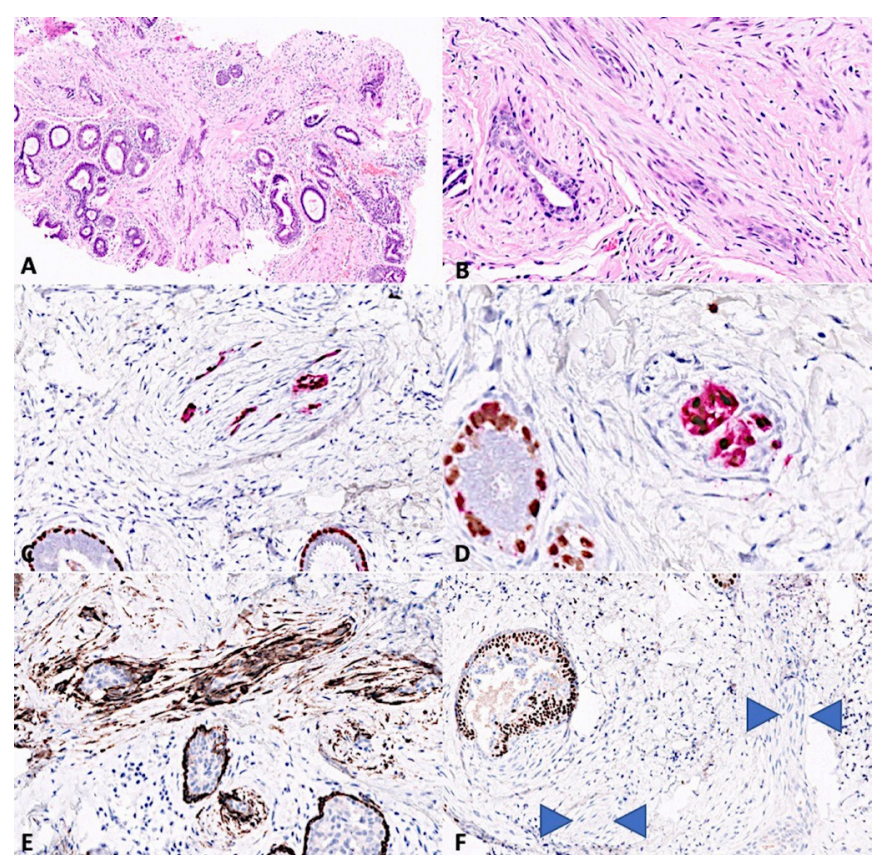

Figure 1 Early radial scar/complex sclerosing lesion. (A) Adenosqamous proliferation (ASP) in area of fibrocystic change (H\&E low-power overview). (B) Detail of ASP (H\&E medium power). (C) Coexpression of CK5/6 and p63 by ASP (CK5 (red)/p63 (brown) dual staining - medium power). (D) Coexpression of CK5/6 and p63 by ASP (CK5 (red)/p63 (brown) dual staining-medium power). (E) Myosin heavy chain - muddy blurry staining of desmoplastic reaction. (F) ASP is oestrogen negative (between arrowheads).

In histological and electron microscopy studies of RSs, Anderson and Battersby ${ }^{39} 40$ suggested that RSs were progressive lesions that could be divided into early and late forms. The descriptions of RSs provided correlates with early (ASP-rich, fibro-elastosis poor) and late (ASP poor/absent, fibro-elastosis rich) RS/CSL. ${ }^{1}$ Histologically, early RS/CSL are characterised by a nidus containing ASP set within loose mucoid stroma with invariably chronic inflammatory cells, with a peripheral corona comprising elements of fibrocystic disease, often with UDH. Small papillomata may occur. There may be an ill-defined nidus or multiple coalescing niduses and a nidus may form a tumour-like focus mimicking a miniature LGASC; the distinction from the latter may be difficult, but the proliferation is typically regarded as benign if it remains confined to the nidus and does not permeate significantly beyond the corona and/or into adipose tissue. In time the ASP appear to involute and the nidus contains residual ducts with an often-incomplete myoepithelial or myoepithelial-like layer within fibroelastosis. Ductal scars may also be apparent. The end result (late RS/CSL) is an often stellate-shaped, 'washed out' fibroelastotic scar with the surrounding corona variably remaining. ${ }^{1}$

RS/CSL can be associated with neoplasia, typically in-situ and/ or invasive ductal or lobular carcinoma that may arise directly within the corona. Small RS $(<0.5 \mathrm{~cm})$ are unlikely to be associated with an adjacent carcinoma. ${ }^{41}$ RS/CSL rarely arise in the absence of proliferative breast disease (PBD) and the increased risk for carcinoma (up to twofold) is generally attributable to the category of coexistent PBD. ${ }^{42}$ The scar-like architecture is therefore almost incidental to the formation of common forms of neoplasia. ${ }^{43}$ The risk of disease 'upgrade' at excision following a diagnosis of RS/CSL depends on the presence or absence of an associated high-risk lesion (HRL) - such as atypical ductal 
hyperplasia (ADH), ductal (DCIS) or lobular carcinoma in-situ (LCIS). The upgrade rate (from core to excision) is reported to lie between $1 \%$ and $3 \%$ with no associated HRL and $1 \%-14 \%$ in the presence of an HRL. Surgical excision (which could include vacuum assisted core biopsy) may be recommended for an HRL, size $>1 \mathrm{~cm}$ or when there are discordant histological and mammographical findings, and observation appropriate when there is absence of the latter and when the lesion has been widely sampled, for example, following $\geq 14$-gauge needle core biopsy (NCB) and/or $>12$ core biopsy sampling. ${ }^{36}$ Vacuum assisted core biopsy revealing an RS/CSL is reliable for excluding malignancy when there is no associated atypia, and radiological and histological findings are concordant. In this setting, imaging-based follow-up is appropriate. ${ }^{44}$ Rhaka $e t a l^{45}$ found that the overall upgrade rate to carcinoma on excision following core biopsy of a high-risk RS/CSL was nearly $25 \%$ and was highest for ADH in which the upgrade rate was similar to that of $\mathrm{ADH}$ not occurring in the context of an RS/CSL. They concluded that the management of RS/CSL should be based on a concurrent atypical lesion detected at core biopsy.

RS/CSL, in common with other SL/BLWS and other lesions (eg, papillomata, NA and adenomyoepithelioma), also show an association with low-grade metaplastic carcinoma including LGASC, spindle cell and fibromatosis-like metaplastic carcinoma, ${ }^{24} 26^{29-33}$ particularly LGASC, which is interesting given the similarity of ASP in benign SL and LGASC. ${ }^{127}$

Using microdissection, RS have been shown to harbour areas that are clonal and neoplastic. ${ }^{46}$ Jacobs et al ${ }^{47}$ demonstrated similarities in mRNA expression for several factors involved in vascular development, in RS and carcinoma (but only invasive ductal carcinoma), suggesting a similar disturbance in epithelialstromal interaction is present in RS and invasive breast carcinoma. Wolters $e \mathrm{al}^{48}$ demonstrated activating PIK3CA mutations in $63.6 \%$ of macrodissected RS but stated that the frequency may reflect the morphology of the associated epithelium (eg, UDH which harbours similar mutations) rather than its underlying architecture or context such as an RS or benign papilloma. More recently, NGS of microdissected ASP from cellular, early $\mathrm{RS} / \mathrm{CSL}$ has demonstrated clonality, also in the form of recurrent PIK3CA mutations. ${ }^{27}$

IE is a term introduced by Azzopardi ${ }^{14}$ for a lesion he agreed was likely synonymous with a RS/CSL under its various epithets. The definition of IE has evolved over time, since inception of the term, with Eusebi and Millis ${ }^{49}$ regarding it different to an RS, stating that it differed from the latter in that it is non-stellate and cellular, having an epitheliosis (UDH) component including Helioid bodies (as previously described in UDH by Tavassoli $e t a l^{50}$ ) and a scleroelastic component resembling LGASC (describing ASP). The concept has further evolved with Eberle ${ }^{26}$ reporting that the lesion contains a UDH-like proliferation that lacks peripheral myoepithelial cells, and demonstrating clonality in the form of recurrent PI3K pathway mutations (PIK3CA in seven cases and PIK3R1 in one case) and clonally related IE, DCIS and LGASC in one case. They pointed out that the rarity of diagnosis of IE was due to most pathologists classifying it as an RS/CSL, and that IE may represent the most proliferative end of the spectrum of these lesions. IE is proposed to form a spectrum with TCCRP, which also has a hyperplasia-like phenotype. ${ }^{202151-55}$

\section{SCLEROSING PAPILLOMA}

IP is a common breast lesion, histologically composed of frond forming/branching, intraductal projections of bilayered

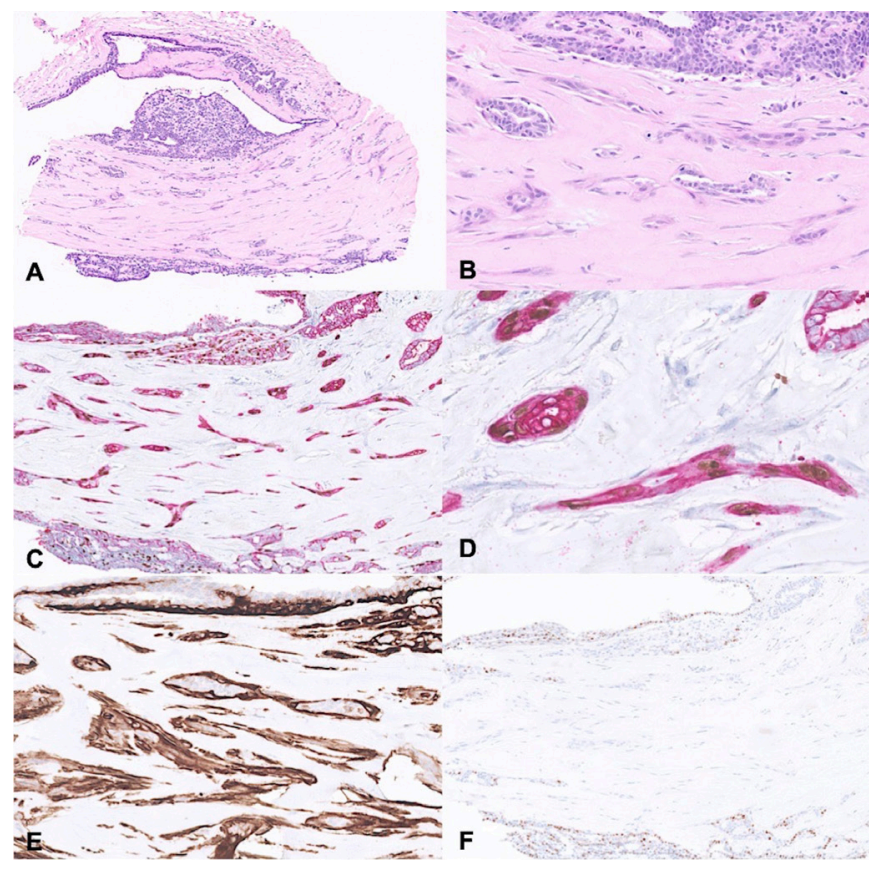

Figure 2 Sclerosing papilloma. (A) Sclerosing papilloma, needle core biopsy (H\&E low-power overview). (B) Adenosquamous proliferation (ASP) with dense sclerotic background (H\&E medium power). (C) Dual CK5 (red) and p63 (brown) immunohistochemistry. (D) Coexpression of CK5 and p63 by ASP (CK5/p63 dual staining-medium power). (E) Myosin heavy chain-muddy blurry staining of desmoplastic reaction. (F) ASP is oestrogen negative.

epithelium and myoepithelium that are supported by stromal cores. They may occur in dilated ducts, cysts ('papillary cystadenoma') or be solid ('DAs'). Various epithelial and myoepithelial changes may be seen including apocrine metaplasia, UDH, myoepithelial hyperplasia, sclerosing changes (SP), lobular and ductal carcinoma in-situ. ${ }^{3656}$ The upgrade rate to cancer from NCB to excision for benign papillomata is between $2 \%$ and $9 \%$, and for papillomata with atypia is $21 \%-37 \%$. Following NCB diagnosis, surgical excision is appropriate for lesions with atypia, and may be appropriate for benign papillomata in individuals $>$ age 55 , lesions $>10 \mathrm{~mm}$, with associated ipsilateral breast cancer or if its upgrade would alter management. ${ }^{36} \mathrm{~A}$ proportion of IP harbour PIK3CA/AKT pathway mutations (figure 2). ${ }^{57} 58$

Sclerosing changes in a papilloma (SP) are not uncommon and there is morphological and immunohistochemical overlap with other SL/BLWS. Their overlap with RS/CSL is documented and they have previously been considered a single entity. ${ }^{520}$ Sclerosis distorts and may obliterate the papilloma in time. Myoepithelium may be markedly attenuated to absent and squamous metaplasia noted. The infiltrative appearance, within and/or at the periphery of the papilloma may mimic low-grade metaplastic carcinoma. ${ }^{205659}$ ASP is a feature of SPs and its presence seems less apparent in more sclerotic, distorted lesions, similar to the evolution seen with RS/CSL. It may be seen within the core of the papilloma or at its base. If carcinoma cannot be excluded, excision biopsy would typically be performed.

\section{DA: WITH SCLEROSIS}

DA is a rare tumour that usually presents in the sixth decade of life as a solitary palpable mass, but it may be multiple. ${ }^{60}$ Although regarded as a distinct lesion by the WHO ${ }^{60}$ it shows considerable overlap with various other SL/BLWS. Histologically, it resembles 


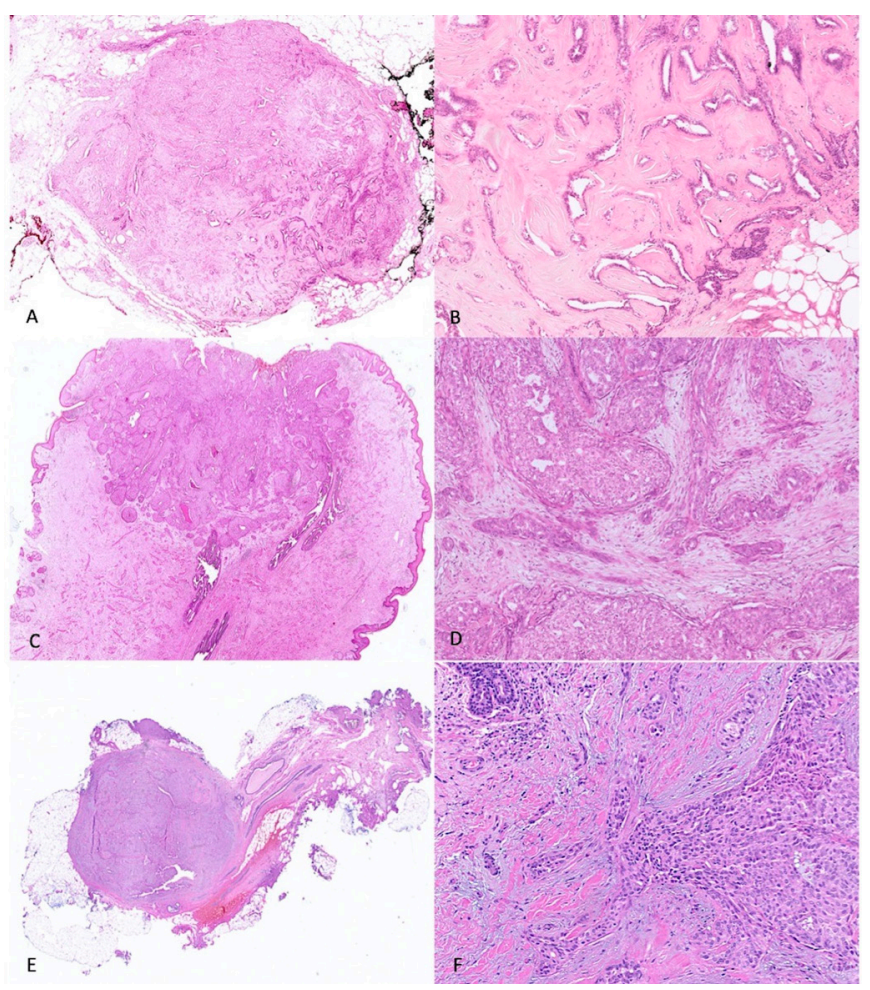

Figure 3 Breast lesions with sclerosis - other. (A) Ductal adenoma (H\&E full section scan). (B) Ductal adenoma-sclerosing architecture (H\&E medium-power). (C) Nipple adenoma (H\&E full section scan). (D) Nipple adenoma-sclerosing architecture with adenosquamous proliferation visible (H\&E full section scan). (E) Subareolar sclerosing duct hyperplasia (H\&E full section scan). (F) Subareolar sclerosing duct hyperplasia—sclerosing architecture (H\&E medium-power).

an IP devoid of the typical arborescent fronded architecture, and resides within a fibrous walled ductal space, of variable calibre, or appears to have outgrown one. Lesions may be single or multifocal, may resemble SP and RS/CSL, lesions sharing the presence of ASP, and can be associated with duct ectasia. ${ }^{1861} \mathrm{In}$ view of mixed histological features in DA, Page and Anderson ${ }^{62}$ regarded them as 'muddling lesions' and in their day-to-day practice reported them as 'sclerosing and adenotic variants of papilloma, nodular sclerosing adenosis or CSL, depending on which they most resemble'. Missense mutations of AKT1, GNAS and PIK3CA have been previously demonstrated. ${ }^{63}$ It measures $0.5-5 \mathrm{~cm}$ (average $0.82 \mathrm{~cm}$ ) and excision is the generally recommended treatment due to mimicry of carcinoma-clinically, radiologically and on core biopsy. Reported associations with malignancy may be coincidental rather than representing malignant transformation (figure $3 \mathrm{~A}, \mathrm{~B}){ }^{60}$

\section{NA: SCLEROSING PAPILLOMATOSIS PATTERN}

$\mathrm{NA}$ is the $\mathrm{WHO}^{64}$ recognised term for the lesion described in 1955 by Jones, ${ }^{65}$ as 'FP of the nipple ducts', typically clinically presenting with nipple enlargement, erythema, erosion and/ or discharge and corresponding histologically to a polymorphous pattern of papillary and adenomatous growth. The same entity has previously been called adenoma of the nipple ${ }^{66}$ and is not the same entity as syringomatous adenoma of the nipple (currently termed ST). ${ }^{67}$ Rosen and Caicco ${ }^{22}$ described four relatively distinct categories, in three, one feature was dominant or exclusively present and in the fourth there were mixed features. Categories described were: sclerosing papillomatosis pattern, papillomatosis (papilloma and UDH) pattern, adenosis pattern and mixed proliferative pattern. Apocrine cysts and squamous cysts are common features of all subtypes. The sclerosing papillomatous pattern of NA comprises ASP and/or sclerosis associated with papillomatous change, and is most likely to be confused with other SL/BLWS, including ST or the rare occurrence of LGASC in the nipple (although the two latter lesions may indeed be synonymous). ${ }^{24} 25$ Similar to other benign proliferative and sclerosing breast lesions, activating PIK3CA mutations are very common in NA and important in its pathogenesis. In addition, KRAS and BRAF mutations may also be important. ${ }^{68}$ Lesions are most common in the fifth decade, range from $0.5 \mathrm{~cm}$ to $>4 \mathrm{~cm}$ and may recur if incompletely excised (figure 3C,D). ${ }^{64}$

\section{SUBAREOLAR SCLEROSING DUCT HYPERPLASIA}

SSDH was described by Rosen $^{23}$ for a central or subareolar lesion, equivalent to NA/FP occurring in the nipple or RS/ CSL occurring in peripheral breast tissue. It was regarded as a benign lesion of which excision alone was adequate treatment. It is essentially a form of SP. Clinical presentation is as a firm or hard tumour, beneath the nipple and/or areola or close to the areola, which may be accompanied by nipple discharge and/or nipple retraction. Lesions average $1.2 \mathrm{~cm}(0.3-3 \mathrm{~cm})$ in size with a broad age range (average around 60 year). The histology is of a sclerosing papillary lesion with moderate UDH and desmoplasia within and surrounding the lesion with entrapped glandular elements with a variable infiltrative appearance. Focal keloidlike stroma and diffuse fibroelastosis are described as well as apocrine metaplasia and squamous metaplasia. At the periphery, CK5/6 expression may be more prominent and myoepithelial cells focally not demonstrable. The description essentially relates to the presence of ASP and associated sclerosis. Significant coexisting lesions within SSDH may include LGASC, DCIS, LCIS and $\mathrm{ADH}$. Recurrence is not recorded after excision. ${ }^{236^{69-71}}$ It is not a specific WHO recognised entity and in day-to-day practice may be reported as a variant of NA, SP or RS/CSL dependent on the predominant histological feature (figure $3 \mathrm{E}, \mathrm{F}$ ).

\section{SYRINGOMATOUS TUMOUR AND LOW-GRADE ADENOSQUAMOUS CARCINOMA}

$\mathrm{ST}$ is the $\mathrm{WHO}^{72}$ recognised term for the entity Rosen ${ }^{67}$ introduced as syringomatous adenoma of the nipple. It is likely included in previous series by Handley and Thackray ${ }^{66}$ and Doctor et $a l .^{73} \mathrm{ST}$ is rare and is regarded as a locally infiltrative, nipple-based counterpart of the also rare LGASC, which typically arises within breast parenchyma. They may measure $0.5 \mathrm{~cm}$ to $>4 \mathrm{~cm}$. Local recurrence may follow incomplete excision. ${ }^{72}$ They are rarely metastasizing, ${ }^{74}$ and can present bilaterally and be locally destructive. ${ }^{75}$ Distinguishing features from LGASC may include a less common occurrence of neoplastic spindle cells, less densely cellular stroma and lack of peripheral lymphoid aggregates (figure 4). ${ }^{41}$

LGASC was described by Rosen and Ernsberger ${ }^{76}$ and the original series of 11 cases was expanded and followed up by Van Hoeven et al. ${ }^{29}$ They demonstrate local infiltration and rarely metastasise, generally when large $(>3 \mathrm{~cm})$, following multiple recurrence or high-grade transformation. LGASC has a distinctive histological appearance and comprises variably compressed tubules that may be lined by columnar or squamous cells with a variable outer layer of myoepithelial-like cells that may merge with squamous elements and may form tadpole-like/syringoid extensions, set within a desmoplastic background that may contain lymphoid aggregates. ${ }^{129334176}$ The age at presentation 


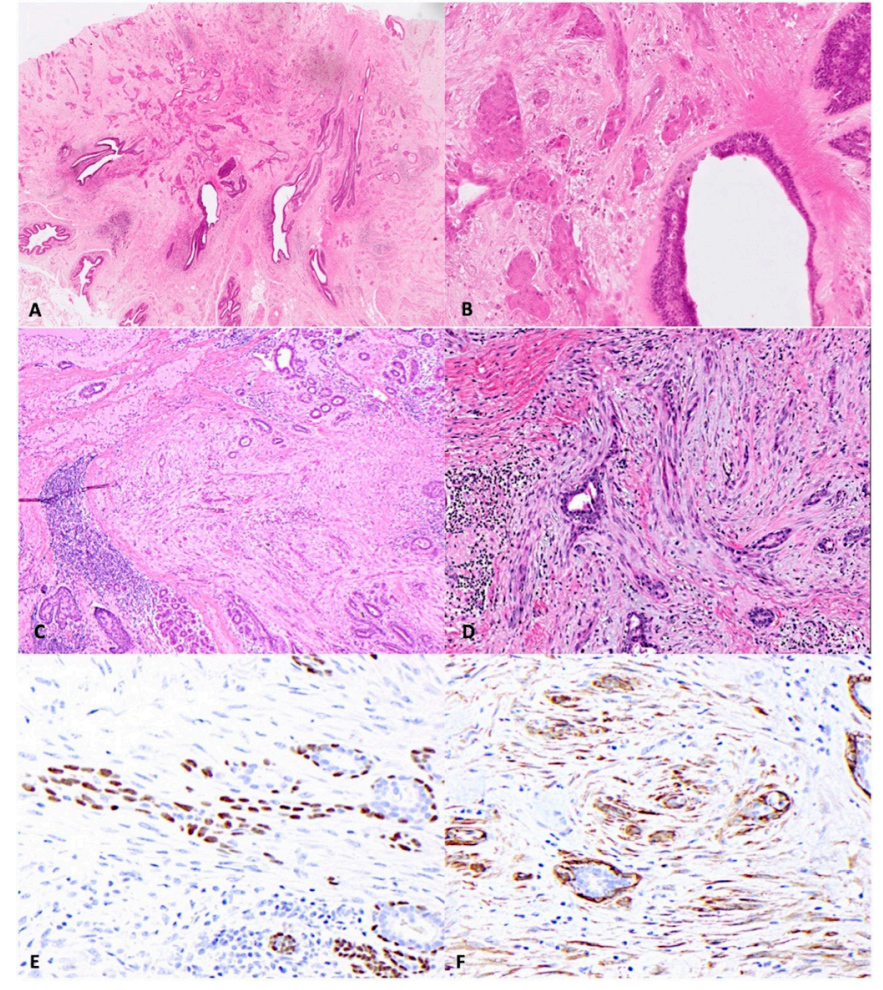

Figure 4 Syringomatous tumour of the nipple (ST) and low-grade adenosquamous carcinoma (LGASC). (A) ST. Skin is absent (top) (H\&Elow power overview scan). (B) Adenosquamous proliferation (ASP)-type invasive tumour predominates - permeating in between lactiferous ducts (H\&E low-power). (C) LGASC. Note lymphoid aggregate on left (H\&E low-power). (D) LGASC. ASP-type infiltrating, neoplastic ducts within desmoplastic stroma predominate (H\&E mediumpower). (E) LGASC. Outer cells and squamoid cells variably express p63 (medium power). (F) LGASC. Myoid markers highlight variable myoepitheliallike cells (particularly in transitional areas) and particularly stromal myofibroblasts (calponin-medium power).

is broad (19-85 years) and size varies from $0.5 \mathrm{~cm}$ to $80 \mathrm{~cm}$, but is mainly $<5 \mathrm{~cm}$. $^{1}$

The immunohistochemical profile of LGASC is characteristic, but not unique. ${ }^{12533417778}$ Geyer et al ${ }^{78}$ demonstrated that LGASC is characterised by complex genetic profiles, including epidermal growth factor receptor (EGFR) amplification. One case has previously occurred in a BRCA1 germline carrier. ${ }^{79}$ It has been shown to harbour PI3K pathway mutations, typically PIK3CA, ${ }^{26} 27$ but not TP53 mutations. ${ }^{28}$

LGASC are often noted in association with benign breast lesions, most typically SL/BLWS. ${ }^{24} 26$-33 Benign ASP-rich SL/ BLWS seem to form a morphological spectrum with LGASC. Morphology is most helpful to distinguish a benign SL/BLWS (eg, RS/CSL) from LGASC such as extent of ASP and degree of infiltration, for example, in an RS/CSL-like lesion, invasion of ASP beyond the corona into breast parenchyma or within predominant desmoplastic stroma. ${ }^{1} 241$ In reference to RS/ CSL, immunohistochemical studies are non-discriminatory and lymphocytic infiltration is common in early proliferative phase lesions, although perhaps not as prominent lymphoid aggregates as described in LGASC. ${ }^{1}$ LGASC may indeed represent an indolent carcinoma whose non-obligate precursor may be identified in RS/CSL, at least in a subset of cases. ${ }^{28}$
The distinction between ST and LGASC is controversial with seemingly histologically identical lesions called benign if located in the nipple region, and malignant if arising within breast parenchyma. Moreover, LGASC has also been reported in a pure nipple location. ${ }^{24}$ Indeed, in a detailed study, Boecker et $a l^{25}$ provided evidence supporting that they are one in the same lesion usually occurring in different locations. As Kanthan and Senger $^{80}$ state 'a single entity with two homes'. Boeckeret al ${ }^{25}$ stated that ST and LGASC were identical or near identical lesions and contained p63, CK5 and CK14 positive $(+)$ cells (generally at the periphery of cell groups) from which a (CK10 and p63 positive) squamous lineage and a $(\mathrm{CK} 8 / 18+, \mathrm{p} 63-)$ glandular lineage arise. Immunohistochemically, identical progenitor cells $(\mathrm{p} 63+/ \mathrm{CK} 5+/ \mathrm{CK} 14+)$ were demonstrated in normal breast duct epithelium of the nipple and/or large breast ducts, supporting that ST and LGASC might both arise from such progenitor cells and both be of breast duct origin (as opposed to ST alternatively being of sweat duct origin). Histologically, both ST and LGASC showed both a luminal glandular and squamous immunophenotype, and an outer myoepithelial-like layer expressing only p63, CK5 and CK14. Myoepithelial differentiation was defined by expression of p63 and/or the basal keratins CK5 and CK14, and myoepithelial marker SMA. Both ST and LGASC were associated with other lesions in some cases, with ST associated with either a papilloma or 'adenomatous adenoma' and LGASC associated with sclerosing adenosis or IP. Occasional myoepithelial cells were seen in ST and LGASC in cases associated with benign lesions, and transitional areas apparent. In summary they stated that in contrast to reports in the literature, the presence of lymphoid aggregates was not found to be a distinguishing feature between ST and LGASC, and myoepithelial markers such as SMA were not constitutively expressed. An encompassing term 'syringomatous adenomatous tumour' may be appropriate. ${ }^{25}$ Both lesions are similarly adequately treated by wide local excision ensuring clear margins.

\section{DISTINCTION BETWEEN LESIONS}

Some of the lesions described lesions are WHO recognised entities, including RS/CSL, NA and DA. Lesions such as IP and NA as entities, are not categorised as SL, but have a sclerosing variant/ subtype with sclerosis (BLWS). In common, they share an association with ASP, which likely is the cause of sclerosis and distortion of the 'base' lesion. Distinguishing between a SL/BLWS with prominent ASP and a LGASC is challenging, and their distinction is subjective. However, a lesion may be best regarded as a LGASC when the ASP forms a distinct tumoural mass with ASP in a pure desmoplastic stroma and moreover when the latter prominently invades adipose tissue. Despite overlapping features, diagnosis rests on a combination of anatomical site and predominant histological feature. ${ }^{27} \mathrm{~A}$ basic outline of lesions and distinguishing features are listed in table 1. A tree diagram aiding distinction of lesions is provided (figure 5).

\section{CONCLUSIONS}

SL/BLWS of the breast form a diverse array of lesions, and often appear histologically similar to one another with the final diagnosis determined by anatomical location or predominant histological feature. A common thread is the sclerosing component, which appears to be secondary at least in most instances, to a common element of ASP, the associated desmoplasia of which appears to distort and modify the base lesion (fibrocystic disease, papilloma, UDH/UDH-like proliferation, etc). The ASP, as is classically seen in RS/CSL, seems to involute and disappear over 


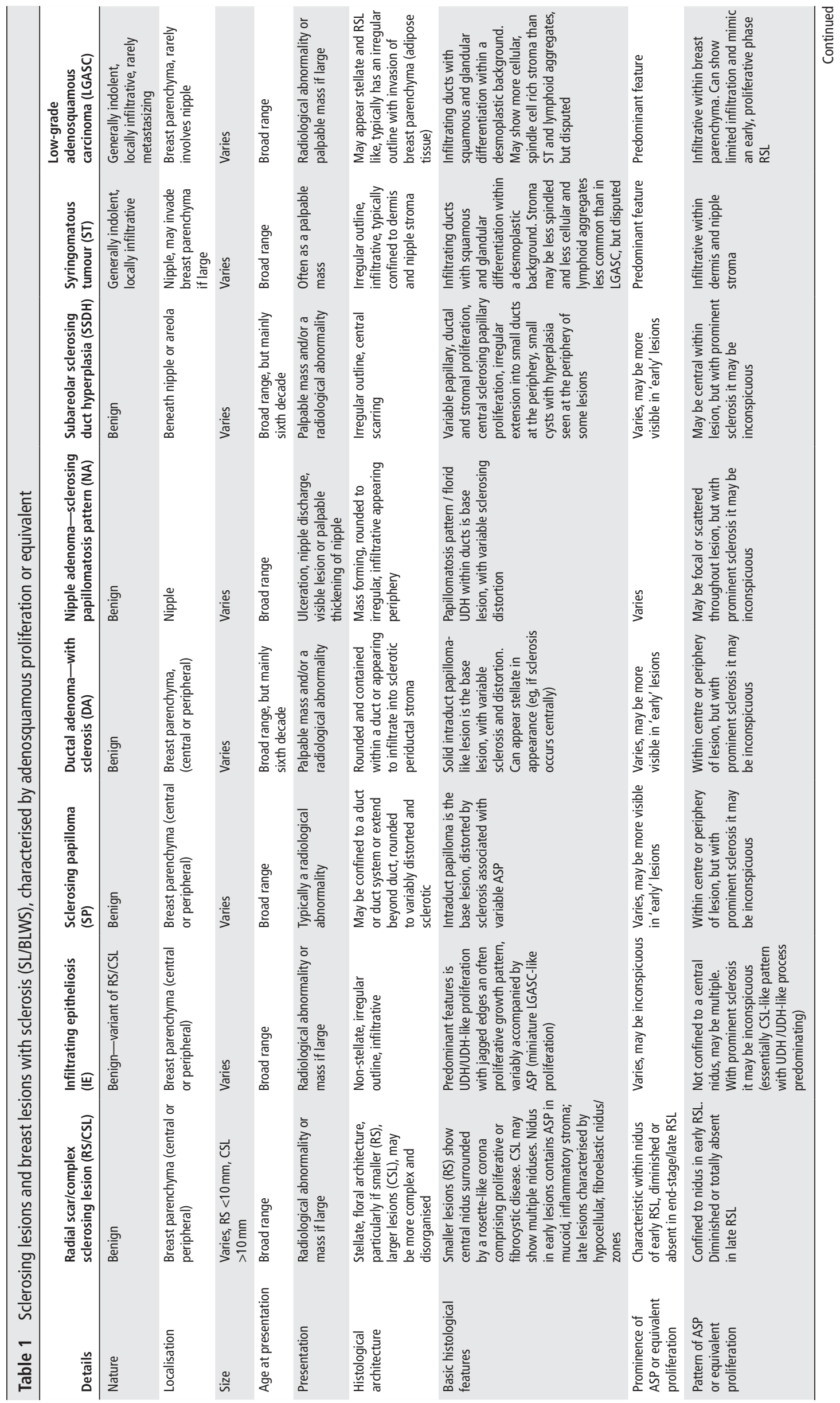




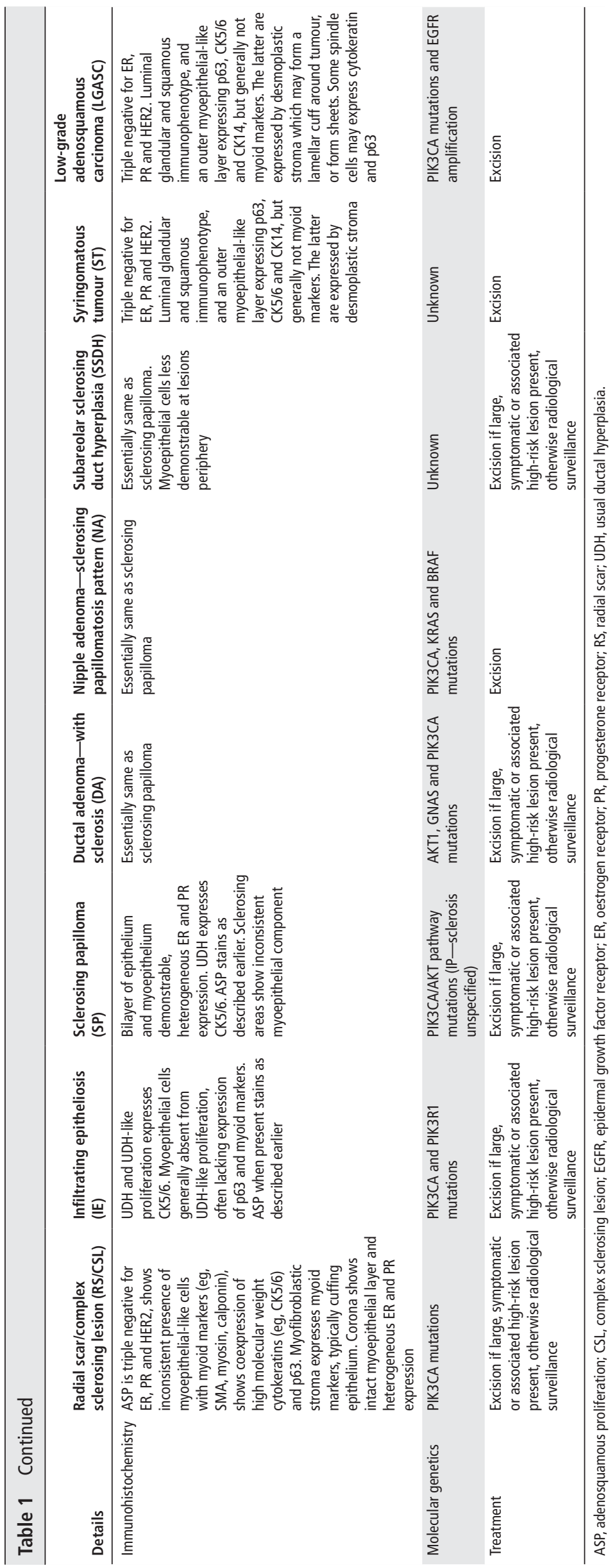

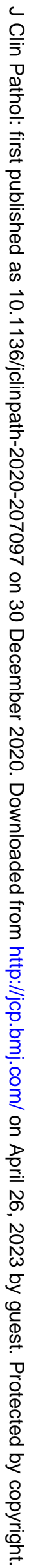




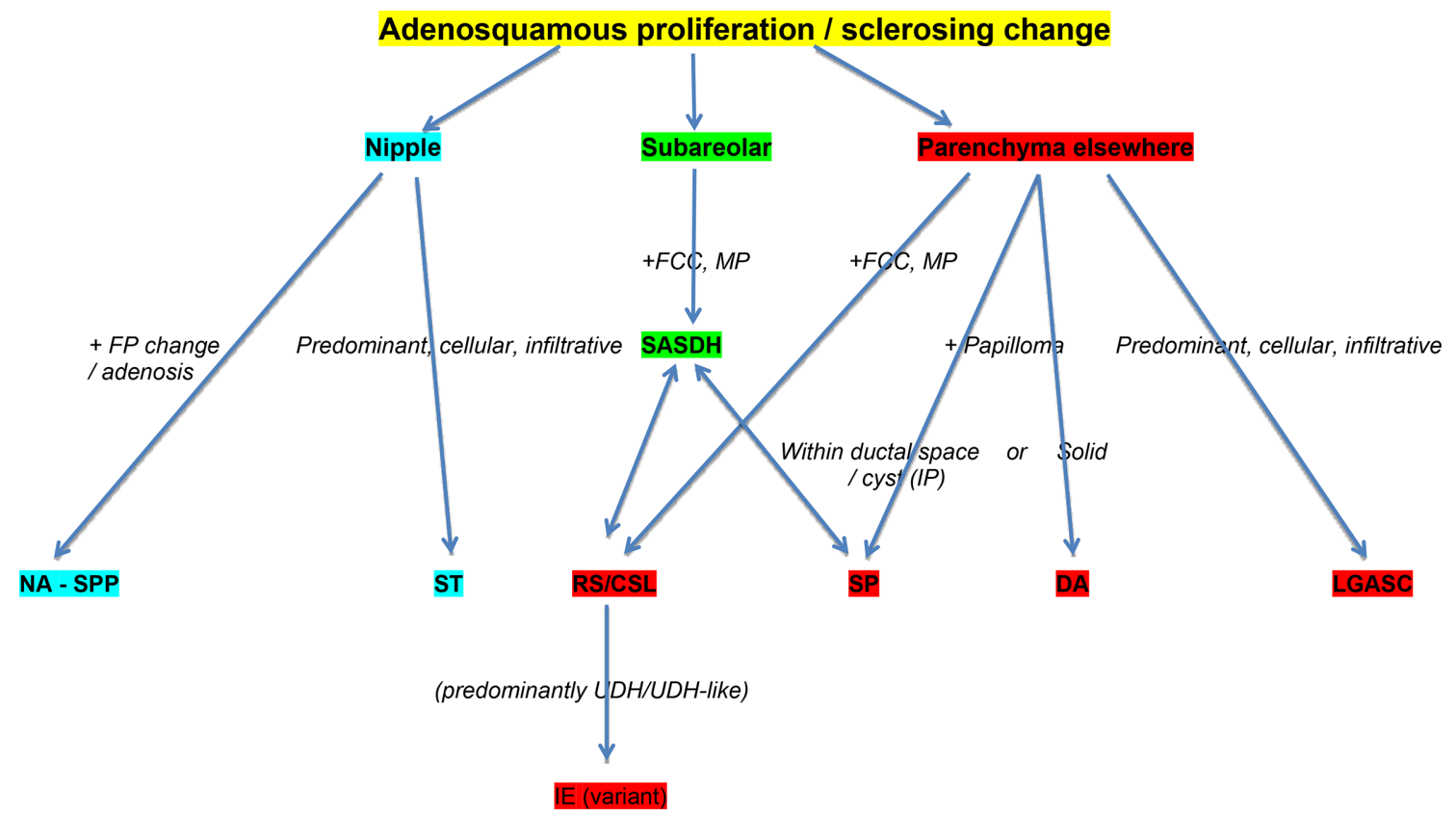

Figure 5 Aide memoir for the classification of sclerosing lesions/breast lesions with sclerosis (SL/BLWS). DA, ductal adenoma; FCC, fibrocystic change; FP, florid papillary change/'papillomatosis'; IE, infiltrating epitheliosis; IP, intraduct papilloma; LGASC, low-grade adenosquamous carcinoma; MP, micropapilloma; NA-SPP, nipple adenoma-sclerosing papillomatosis pattern; RS/CSL, radial scar/complex sclerosing lesion; SASDH, subareolar sclerosing duct hyperplasia; SP, sclerosing papilloma; ST, syringomatous tumour; UDH, usual ductal hyperplasia.

time. It may be a prominent to inconspicuous, or even absent component in any one lesion at the time of histological assessment. SL/BLWS have an inherent neoplastic risk with 'common' carcinomas (in-situ or invasive) arising from the non-scleroelastic component (UDH, etc) due to its inherent neoplastic risk regardless of its association with a sclerosing lesion and low-grade metaplastic carcinoma, particularly LGASC, perhaps derived from persistent growth of ASP characterising the sclerosing component. Distinction between the various entities may be difficult and any one lesion may fit several categories. Difficultto-categorise lesions are typically adequately treated by local excision with clear margins.

\section{Take home messages}

- Adenosquamous proliferation of the breast is a key component of sclerosing lesions and breast lesions with sclerosis.

- ASP in SL/BLWS is phenotypically similar to the neoplastic proliferation characteristic of syringomatous tumour and lowgrade adenosquamous carcinoma.

- ASP may represent a non-obligate neoplastic precursor lesion for low-grade metaplastic carcinoma, eg, LGASC.

- Distinguishing between SL/BLWS with prominent ASP and LGASC can be diagnostically challenging and their distinction may be based on the extent of the ASP.

\section{Handling editor Cheok Soon Lee.}

Acknowledgements Thanks to Lucia Simone, Elaine Borg and LGT library for sourcing referenced articles. Thanks to David Allen and Luis Campos for immunohistochemical studies. Thanks for the following cases and/or images: Sami Shousha (Figures 3A-D and 4A,B), Elizabeth Sinclair (Figure 3E-F).

Contributors I am the sole author of this article.

Funding The author has not declared a specific grant for this research from any funding agency in the public, commercial or not-for-profit sectors.
Competing interests None declared.

Patient consent for publication Not required.

Provenance and peer review Not commissioned; externally peer reviewed.

ORCID iD

Mark James Wilsher http://orcid.org/0000-0002-7959-9671

\section{REFERENCES}

1 Wilsher MJ. Adenosquamous proliferation of the breast and low grade adenosquamous carcinoma: a common precursor of an uncommon cancer? Pathology 2014;46:402-10.

2 Agrawal A, Saha S, Ellis 10, et al. Adenosquamous carcinoma of breast in a 19 years old woman: a case report. World J Surg Oncol 2010;8:44.

3 Hamperl H. Radial scars (scarring) and obliterating mastopathy. Virchows Arch A Pathol Anat Histol 1975;369:55-68.

4 Linell F, Ljungberg 0, Andersson I. Breast carcinoma. aspects of early stages, progression and related problems. Acta Pathol Microbiol Scand Suppl 1980;272:1-233.

5 Fenoglio C, Lattes R. Sclerosing papillary proliferations in the female breast. A benign lesion often mistaken for carcinoma. Cancer 1974;33:691-700.

6 Semb CB. Pathologico-anatomical and clinical investigations of fibroadenomatosiscystica mammae, and its relation to other pathological conditions in the mamma, especially cancer. Acta Chir Scand 1928;64:1-484.

7 D'Amore E, Montes E, Le MG, et al. [Aschoff's center of proliferation. Experience of the Gustave Roussy Institute]. Ann Pathol 1985;5:173-82.

8 Schaper A. Beiträge Zur analyse des tierischen Wachstums. Archiv für Entwicklungsmechanik der Organismen 1905;19:348-445.

9 Borst M. Das pathologische Wachtum. In: Aschoff L, ed. Pathologische Anatomie. Erster band: Allgemeiner Teil. Jena: Gustav Fischer, 1911: 555-738.

10 McDivitt RW, Stewart FW, Berg LW. Benign breast lesions. In: Tumors of the breast, AFIP atlas of tumor pathology. . 2nd Series, 1968: 2. 133-49.

11 Eusebi V, Grassigli A. Grosso F: Lesioni focali scleroelastotiche mammarie simulanti IL carcinoma infiltrante. Pathologica 1976;68:507-18.

12 Tremblay G, Buell RH, Seemayer TA. Elastosis in benign sclerosing ductal proliferation of the female breast. Am J Surg Pathol 1977;1:155-60.

13 Fisher ER, Palekar AS, Kotwal N, et al. A nonencapsulated sclerosing lesion of the breast. Am J Clin Pathol 1979;71:240-6.

14 Azzopardi JG. Overdiagnosis of malignancy. In: Problems in breast pathology. London: WB Saunders, 1979: vol II. 167-90.

15 Rickert RR, Kalisher L, Hutter RV. Indurative mastopathy: a benign sclerosing lesion of breast with elastosis which may simulate carcinoma. Cancer 1981;47:561-71. 
16 Page OL, Anderson TJ. Radial scars and complex sclerosing lesions. In: Diagnostic histopathology of the breast. Edinburgh: Churchill Livingstone, 1987: 89-103.

17 Koerner F. Papilloma and related benign tumours. In: Rosen's Breast Pathology. 4th edn. Lippincott Williams and Wilkins, 2009: 95-152.

18 Azzopardi JG, Salm R. Ductal adenoma of the breast: a lesion which can mimic carcinoma. J Pathol 1984;144:15-23.

19 Yang WT, Bu H, Foschini MP, et al. Tall cell carcinoma with reversed polarity. breast tumours, edited by who classification of tumours editorial board. 5th edn. Lyon: WHO Classification of Tumours Editorial board, 2019: 153-4.

20 Agoumi M, Giambattista J, Hayes MM. Practical considerations in breast papillary lesions: a review of the literature. Arch Pathol Lab Med 2016;140:770-90.

21 Bhargava R, Florea AV, Pelmus $\mathrm{M}$, et al. Breast tumor resembling tall cell variant of papillary thyroid carcinoma: a solid papillary neoplasm with characteristic immunohistochemical profile and few recurrent mutations. Am J Clin Pathol 2017:147:399-410

22 Rosen PP, Caicco JA. Florid papillomatosis of the nipple. A study of 51 patients, including nine with mammary carcinoma. Am J Surg Pathol 1986;10:87-101.

23 Rosen PP. Subareolar sclerosing duct hyperplasia of the breast. Cancer 1987:59:1927-30.

24 Wilsher MJ, Desai AJ, Pinder SE. Low-grade adenosquamous carcinoma arising in association with a nipple adenoma. Histopathology 2020;76:784-7.

25 Boecker W, Stenman G, Loening T, et al. Differentiation and histogenesis of syringomatous tumour of the nipple and low-grade adenosquamous carcinoma: evidence for a common origin. Histopathology 2014;65:9-23.

26 Eberle CA, Piscuoglio S, Rakha EA, et al. Infiltrating epitheliosis of the breast: characterization of histological features, immunophenotype and genomic profile. Histopathology 2016;68:1030-9.

27 Wilsher MJ, Owens TW, Allcock RJ. Next generation sequencing of the nidus of early (adenosquamous proliferation rich) radial sclerosing lesions of the breast reveals evidence for a neoplastic precursor lesion. J Pathol Clin Res 2017;3:115-22.

28 Bataillon G, Fuhrmann L, Girard E, et al. High rate of PIK3CA mutations but no TP53 mutations in low-grade adenosquamous carcinoma of the breast. Histopathology 2018;73:273-83.

29 Van Hoeven $\mathrm{KH}$, Drudis T, Cranor ML, et al. Low-grade adenosquamous carcinoma of the breast. A clinocopathologic study of 32 cases with ultrastructural analysis. Am J Surg Pathol 1993;17:248-58.

30 Foschini MP, Pizzicannella G, Peterse JL, et al. Adenomyoepithelioma of the breast associated with low-grade adenosquamous and sarcomatoid carcinomas. Virchows Arch 1995;427:243-50.

31 Denley $\mathrm{H}$, Pinder SE, Tan $\mathrm{PH}$, et al. Metaplastic carcinoma of the breast arising within complex sclerosing lesion: a report of five cases. Histopathology 2000;36:203-9.

32 Gobbi H, Simpson JF, Jensen RA, et al. Metaplastic spindle cell breast tumors arising within papillomas, complex sclerosing lesions, and nipple adenomas. Mod Pathol 2003;16:893-901.

33 Soo K, Tan PH. Low-grade adenosquamous carcinoma of the breast. J Clin Pathol 2013;66:506-11.

34 Suster S, Moran CA, Hurt MA. Syringomatous squamous tumors of the breast. Cancer 1991;67:2350-5.

35 Sahin A, Collins LC. Radial scar / complex sclerosing lesion. breast tumours, edited by WHO classification of tumours editorial board. 5th edn. Lyon: WHO Classification of Tumours Editorial board, 2019: 30-1.

36 Racz JM, Carter JM, Degnim AC. Challenging atypical breast lesions including flat epithelial atypia, radial scar, and intraductal papilloma. Ann Surg Oncol 2017:24:2842-7.

37 Azzopardi JG. Elastosis and other connective tissue changes. In: Problems in breast pathology. London: WB Saunders, 1979: vol II. 379-94.

38 Parfrey NA, Doyle CT. Elastosis in benign and malignant breast disease. Hum Pathol 1985;16:674-6.

39 Anderson TJ, Battersby S. Radial scars of benign and malignant breasts: comparative features and significance. J Pathol 1985;147:23-32.

40 Battersby $S$, Anderson TJ. Myofibroblast activity of radial scars. J Pathol $1985 ; 147: 33-40$.

41 Hicks DG, Lester SJ. Benign epithelial lesions. In: Diagnostic pathology: breast. 2nd edn. Elsevier, 2016: 90-181.

42 Sanders ME, Page DL, Simpson JF, et al. Interdependence of radial scar and proliferative disease with respect to invasive breast carcinoma risk in patients with benign breast biopsies. Cancer 2006;106:1453-61.

43 Alvarado-Cabrero I, Tavassoli FA. Neoplastic and malignant lesions involving or arising in a radial scar: a clinicopathologic analysis of 17 cases. Breast J 2000;6:96-102.

44 Bacci J, MacGrogan G, Alran L, et al. Management of radial scars/complex sclerosing lesions of the breast diagnosed on vacuum-assisted large-core biopsy: is surgery always necessary? Histopathology 2019;75:900-15.

45 Rakha E, Beca F, D'Andrea M, et al. Outcome of radial scar/complex sclerosing lesion associated with epithelial proliferations with atypia diagnosed on breast core biopsy: results from a multicentric UK-based study. J Clin Pathol 2019;72:800-4.

46 Iqbal M, Shoker BS, Foster CS, et al. Molecular and genetic abnormalities in radial scar. Hum Pathol 2002;33:715-22.
47 Jacobs TW, Byrne C, Colditz G, et al. Radial scars in benign breast-biopsy specimens and the risk of breast cancer. N Eng/ J Med 1999;340:430-6.

48 Wolters KL, Ang D, Warrick A, et al. Frequent PIK3CA mutations in radial scars. Diagn Mol Pathol 2013:22:210-4.

49 Eusebi V, Millis RR. Epitheliosis, infiltrating epitheliosis, and radial scar. Semin Diagn Pathol 2010:27:5-12.

50 Tavassoli FA, Majeste RM, Snyder RC. Intranuclear helioid inclusions in mammary intraductal hyperplasias. Ultrastruct Pathol 1991;15:267-79.

51 Eusebi V, Damiani S, Ellis IO, et al. Breast tumor resembling the tall cell variant of papillary thyroid carcinoma: report of 5 cases. Am J Surg Pathol 2003;27:1114-8.

52 Lozada JR, Basili T, Pareja F, et al. Solid papillary breast carcinomas resembling the tall cell variant of papillary thyroid neoplasms (solid papillary carcinomas with reverse polarity) harbour recurrent mutations affecting IDH2 and PIK3CA: a validation cohort. Histopathology 2018;73:339-44.

53 Chiang S, Cole KS, Baker G, et al. Solid papillary neoplasms with reverse polarization (SPNRPs): histologic, immunophenotypic and genotypic characterization of a distinctive breast tumor [abstract 155]. Mod Pathol 2014:27:4-566.

54 Chiang S, Weigelt B, Wen $\mathrm{H}-\mathrm{C}$, et al. Solid papillary carcinoma with reverse polarization are driven by IDH2 and PIK3 pathway mutations [abstract 138]. Am Assoc Cancer Res 2016.

55 Chang SY, Fleiszer DM, Mesurolle B, et al. Breast tumor resembling the tall cell variant of papillary thyroid carcinoma. Breast J 2009;15:531-5.

56 Koerner FC. Papilloma and related benign lesions. Available: https://basicmedicalkey. com/papilloma-and-related-benign-lesions/

57 Mishima C, Kagara N, Ikeda J-I, et al. Mutational analysis of AKT1 and PIK3CA in intraductal papillomas of the breast with special reference to cellular components. Am J Pathol 2018;188:1106-12.

58 Troxell ML, Levine J, Beadling C, et al. High prevalence of PIK3CA/AKT pathway mutations in papillary neoplasms of the breast. Mod Pathol 2010;23:27-37.

59 Azzopardi JG. Papilloma and papillary carcinoma. In: Problems in breast pathology. London: WB Saunders, 1979: vol II. 150-66.

60 Jaffer SM, Moritani S, Silva L, et al. Breast tumours, edited by who classification of tumours editorial board. 5td edn. Lyon: WHO Classification of Tumours Editorial board, 2019: 37-8.

61 Lammie GA, Millis RR. Ductal adenoma of the breast--a review of fifteen cases. Hum Pathol 1989;20:903-8.

62 Page OL, Anderson TJ. Papilloma and related lesions. In: Diagnostic histopathology of the breast. Edinburgh: Churchill Livingstone, 1987: 104-19.

63 Volckmar A-L, Leichsenring J, Flechtenmacher C, et al. Tubular, lactating, and ductal adenomas are devoid of MED12 Exon2 mutations, and ductal adenomas show recurrent mutations in GNAS and the PI3K-AKT pathway. Genes Chromosomes Cancer 2017:56:11-17.

64 Lester SC, AHS L. Nipple adenoma. Breast tumours, edited by WHO classification of tumours editorial board. 5th edn. Lyon: WHO Classification of Tumours Editorial board, 2019: 182-3.

65 Jones DB. Florid papillomatosis of the nipple ducts. Cancer 1955:8:315-9.

66 Handley RS, Thackray AC. Adenoma of nipple. Br J Cancer 1962;16:187-94.

67 Rosen PP. Syringomatous adenoma of the nipple. Am J Surg Pathol 1983:7:739-45.

68 Liau J-Y, Lee Y-H, Tsai J-H, et al. Frequent PIK3CA activating mutations in nipple adenomas. Histopathology 2017;70:195-202.

69 Tressera F, Monzón M, Gracses PJ, et al. Subareolar sclerosing ductal hyperplasia of the breast: report of eight cases. Rev Senología y Patol Mam 2003;16:31-4.

70 Cheng E, D'Alfonso TM, Arafah M, et al. Subareolar sclerosing ductal hyperplasia. Int Surg Pathol 2017;25:4-11.

71 Gulati A, Kaushal V, Jaswal KS, et al. Subareolar sclerosing duct hyperplasia of the breast: an encounter with a rare entity. Arch Med Health Sci 2017;5:95-6.

72 Lester SC, AHS L. Syringomatous tumour. Breast tumours, edited by WHO classification of tumours editorial board. 5th edn. Lyon: WHO Classification of Tumours Editorial board, 2019: 180-1.

73 Doctor VM, Sirsat MV, Papillomatosis F. Florid papillomatosis (adenoma) and other benign tumours of the nipple and areola. Br J Cancer 1971;25:1-9.

74 Chang CK, Jacobs IA, Calilao G, et al. Metastatic infiltrating syringomatous adenoma of the breast. Arch Pathol Lab Med 2003;127:e155-6.

75 Montgomery ND, Bianchi GD, Klauber-Demore N, et al. Bilateral syringomatous adenomas of the nipple: case report with immunohistochemical characterization of a rare tumor mimicking malignancy. Am J Clin Pathol 2014;141:727-31.

76 Rosen PP, Ernsberger D. Low-grade adenosquamous carcinoma. A variant of metaplastic mammary carcinoma. Am J Surg Pathol 1987;11:351-8.

77 Kawaguchi K, Shin SJ. Immunohistochemical staining characteristics of low-grade adenosquamous carcinoma of the breast. Am J Surg Pathol 2012;36:1009-20.

78 Geyer FC, Lambros MBK, Natrajan R, et al. Genomic and immunohistochemical analysis of adenosquamous carcinoma of the breast. Mod Pathol 2010;23:951-60.

79 Noël J-C, Buxant F, Engohan-Aloghe C. Low-grade adenosquamous carcinoma of the breast--A case report with a BRCA1 germline mutation. Pathol Res Pract 2010;206:511-3.

80 Kanthan R, Senger JL. Low-Grade Adenosquamous Carcinoma [LGASC] of the Breast and Syringomatous Adenoma of the Nipple [SAN]: A Single Entity with Two Homes? Ann Clin Pathol 2014;2:1026. 